\title{
Enhanced fluorescence by metal nanospheres on metal substrates
}

\author{
Stefania D’Agostino, ${ }^{1,2}$ Pier Paolo Pompa, ${ }^{1}$ Rocco Chiuri, ${ }^{1,2}$ Raymond J. Phaneuf, ${ }^{3}$ Dominic G. Britti, ${ }^{3}$ \\ Rosaria Rinaldi, ${ }^{1,2}$ Roberto Cingolani, ${ }^{1}$ and Fabio Della Sala ${ }^{1, *}$ \\ ${ }^{1}$ National Nanotechnology Laboratory of CNR-INFM, Research Unit of the Italian Institute of Technology, \\ I-73100 Lecce, Italy \\ ${ }^{2}$ Instituto Superiore Universitario di Formazione Interdisciplinare, University of Salento, I-73100 Lecce, Italy \\ ${ }^{3}$ Department of Materials Science and Engineering and Laboratory for Physical Science, University of Maryland, \\ College Park, Maryland 20740, USA \\ *Corresponding author: fabio.dellasala@unile.it
}

Received April 8, 2009; revised June 25, 2009; accepted July 7, 2009; posted July 15, 2009 (Doc. ID 109676); published July 31, 2009

\begin{abstract}
We investigate the metal enhanced fluorescence by silver nanospheres on a thin silver substrate. Experimental measurements for core/shell colloidal nanocrystals embedded in a polymer matrix show a fluorescence enhancement factor of about 9. We apply the discrete dipole approximation method to describe the local-field enhancement factor (LFEF). We find that the observed fluorescence enhancement is related to the broad LFEF profile induced by the substrate. (C) 2009 Optical Society of America OCIS codes: $240.6680,050.1755,260.2510$.
\end{abstract}

During the past few years nanotechnology advances have allowed material science to structure and characterize metallic nanostructures and/or nanoparticles with high precision, thus increasing the interest of scientists in surface plasmons [1,2]. Owing to their localized surface plasmon resonances (LSPRs), metal nanoparticles (MNPs) are largely used to enhance the fluorescence of radiators (metal enhanced fluorescence, MEF), such as organic molecules [2,3] or semiconductor nanocrystals (NCs) [4].

In this work we are interested in the description of the plasmon resonances of silver nanospheres touching thin silver substrates. The system under investigation is depicted in Fig. 1.

CdSe/ZnS core/shell colloidal NCs (Evident Technologies, Troy, New York), were dispersed in polymethyl methacrylate (PMMA-950K) (final concentrations in chlorobenzene: $C_{\mathrm{NC}}=7 \times 10^{-6} \mathrm{M}$ and $C_{\mathrm{PMMA}}$ $\left.=1.9 \times 10^{-5} \mathrm{M}\right)$ and deposited by spin coating onto the plasmonic substrates. These consist of $75 \mathrm{~nm} \mathrm{Ag}$ spheres randomly distributed on a 70-nm-thick Ag substrate with a surface coverage of about $10 \%$ and covered by an $\mathrm{Al}_{2} \mathrm{O}_{3}$ film of increasing thickness $(d)$ from 0.5 to $100 \mathrm{~nm}$ (as shown in Fig. 1). The polymeric film, characterized by atomic force microscopy and confocal microscopy, exhibits a good uniformity (with a thickness of $50 \mathrm{~nm}$ ) and a homogeneous distribution of the NCs, with spectral features similar to those of the reference NC samples in the solution [5]. Photoluminescence excitation (PLE) and photoluminescence (PL) measurements $\left(\lambda_{\text {exc }}^{P L}=460 \mathrm{~nm}\right)$ are reported in Fig. 2. The PLE curves show a distinct peak at $460 \mathrm{~nm}$, which seems to be related to the plasmonic substrate, since such spectral features are not present for the reference substrate.

Figure 2 shows that already for the thinner oxide layer a PL enhancement of 6.7 is present. The maximum enhancement (9.2) is obtained for $d=5 \mathrm{~nm}$, while for larger thicknesses it decreases (down to 2.4 for $d=100 \mathrm{~nm}$ ).
The presence of the metal substrate is expected to reduce substantially the luminescence at very short fluorophore-metal distance owing to energy dissipation in the metal $[1,2,6]$. In our case, however, the emitting dipoles (i.e., the NCs) are uniformly distributed in the 50-nm-thick PMMA matrix, which is thus quite far ( $25 \mathrm{~nm}$ on the average) from the substrate. Moreover, previous studies using metal mirror surfaces $[2,6-10]$ have shown that an underlying metal buffer can instead enhance the fluorescence of a radiator owing to the coupling between the LSPR and the surface plasmon. Here, we want to verify the applicability of these substrates for MEF applications.

For a quantitative analysis of these effects, the electromagnetic computation of the local-field enhancement factor (LFEF) [11] for MNPs on metal substrates is required. Despite different approaches that have been proposed [12-14], for arbitrary systems of complex shapes and large dimensions, a fully numerical solution of Maxwell's equations is mandatory. In particular the image-dipole approaches [12] cannot be applied in our case, as the thickness of metal substrate is smaller than the size of the MNP.

The discrete dipole approximation (DDA) $[15,16]$ has attracted a lot of interest, because it allows a

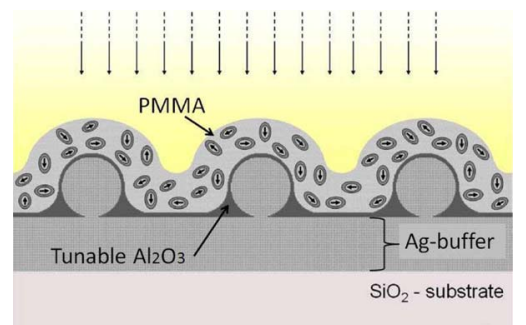

Fig. 1. (Color online) System under investigation: the outer layer represents the 50-nm-thick PMMA film containing the NCs (with an isotropic orientation); the $\mathrm{Ag}$ spheres with a $75 \mathrm{~nm}$ diameter are covered by a tunable $\mathrm{Al}_{2} \mathrm{O}_{3}$ layer and deposited on a $70 \mathrm{~nm} \mathrm{Ag}$ buffer lying on a $\mathrm{SiO}_{2}$ substrate. 


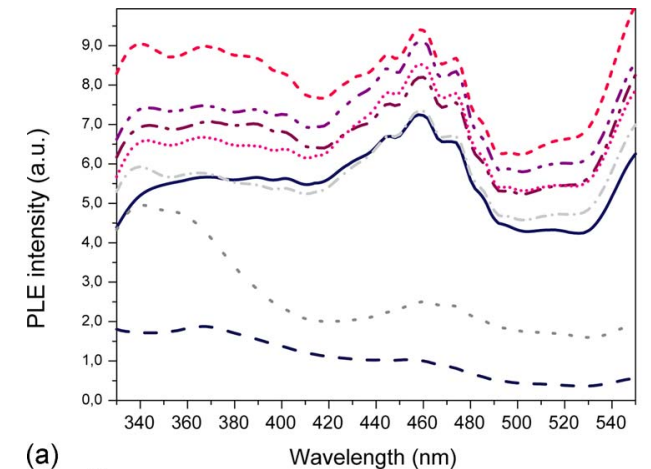

(a)

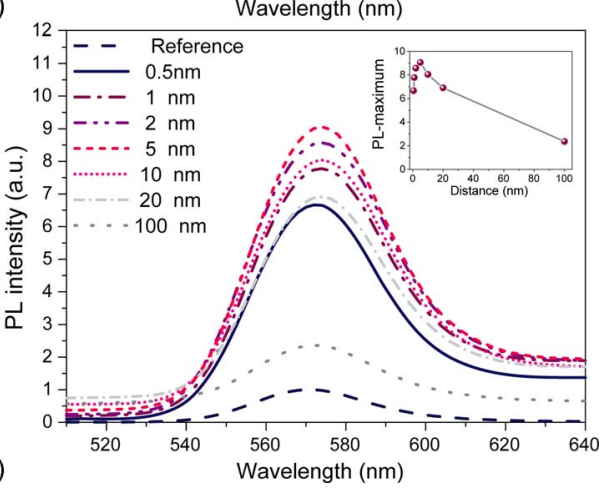

Fig. 2. (Color online) (a) PLE and (b) PL spectra for different oxide thicknesses. The "reference" substrate (with a PL maximum normalized to unity) is without MNPs and the silver layer. Inset in (b): PL maximum versus oxide thickness.

quite fast and accurate calculation of the scattering efficiencies [11]. The substrate effect can be described using a finite-size box $[3,17]$. In the limit of large sizes of the computational box (simulating the infinite plane), such a method gives the exact physical solution to the retarded Maxwell's electromagnetic equations without making any limiting assumptions. A periodic approach is not appropriate here (low coverage and random distribution of MNPs).

Here, we have investigated this system by using the DDA approach as implemented in the Amsterdam discrete dipole approximation (ADDA) code [18]. We discretized jointly the sphere and the substrate by using a finite box of polarizable points. Convergence tests (not reported here) showed that a large substrate of $500 \mathrm{~nm}$ on a side and an interdipole distance of $1 \mathrm{~nm}$ is appropriate to reach the convergence for the extinction and the absorption cross sections, as well as for the $E$-field values. The total system contains almost $18 \times 10^{6}$ dipoles, and simulations have been performed by parallelizing the target on 40 cores (Opteron 2356) occupying 22 Gbytes of total memory. Convergence is achieved in 176 iterations (48 min CPU time). We also considered an isolated silver sphere of the same dimension to directly investigate the effects of the substrate. We used $\lambda_{\text {exc }}$ $=380 \mathrm{~nm}$ (corresponding to the computed plasmon resonance of the isolated sphere) and a uniform incident field propagating normal to the metallic surface and polarized along the $y$ direction. Calculations performed at different excitation wavelengths show similar results as the nanoparticle plasmon resonance is strongly broadened owing to the interaction with the substrate $[2,12]$.

We implemented a parallel routine (not available in ADDA) that is able to compute the total field $\left(\mathbf{E}_{t o t}\right)$ on an arbitrary set of points $\mathbf{r}_{i}$,

$$
\mathbf{E}_{t o t}\left(\mathbf{r}_{i}\right)=\mathbf{E}_{0}\left(\mathbf{r}_{i}\right)+\sum_{k=1, N} \mathbf{G}\left(\mathbf{r}_{k}, \mathbf{r}_{i}\right) \mathbf{P}\left(\mathbf{r}_{k}\right),
$$

where $\mathbf{E}_{0}$ is the incident field, $\mathbf{G}$ is the dyadic Green's function [16], and $\mathbf{P}$ are the polarizations dipoles. The LFEF of the real system is then computed as (assuming $\left|\mathbf{E}_{0}\right|=1$ )

$$
\operatorname{LFEF}(\mathbf{r})=\left|\mathbf{E}_{t o t}^{s y s}(\mathbf{r})-\mathbf{E}_{t o t}^{s u b}(\mathbf{r})+\mathbf{E}_{0}(\mathbf{r})+\mathbf{E}_{r e f}(\mathbf{r})\right|^{2},
$$

where $\mathbf{E}_{t o t}^{s y s}$ is the field of the total system (sphere + substrate), $\mathbf{E}_{t o t}^{s u b}$ is the field of the substrate alone, and $\mathbf{E}_{r e f}=r\left(\omega_{\text {exc }}\right) \mathbf{E}_{0}$ is the field reflected from an (in plane) infinite substrate. Using Eq. (2), spurious boundary effects owing to the finite size of the substrate employed in the ADDA calculation are avoided.

Figure 3 reports the calculated LFEF contours for

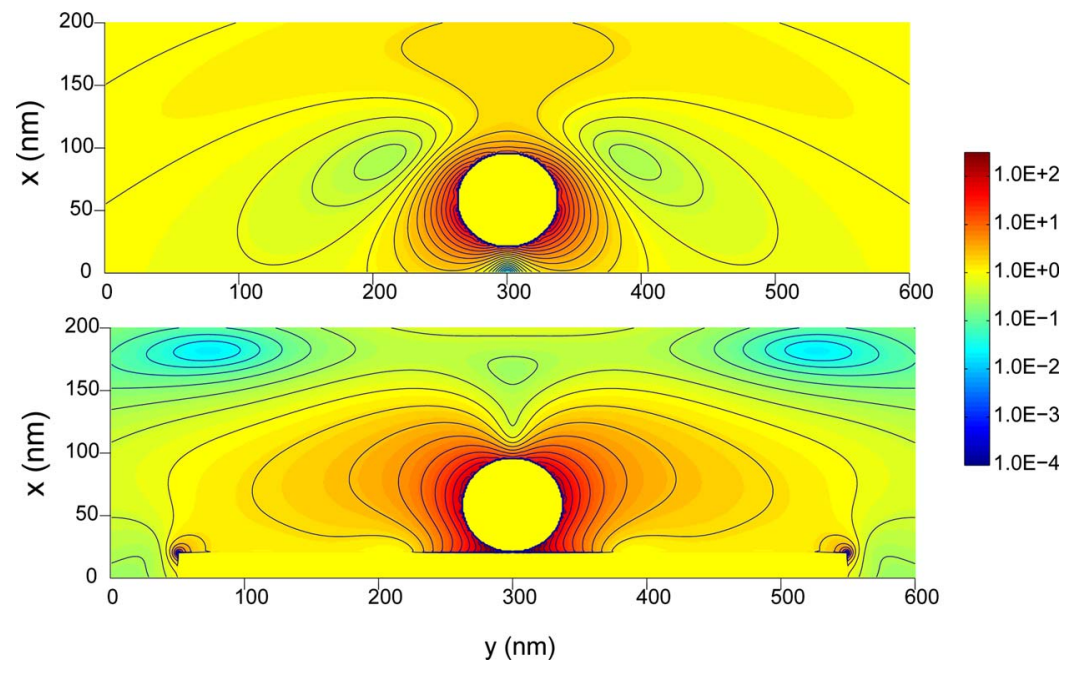

Fig. 3. (Color online) Computed LFEF contours (logarithmic scale) for a $75 \mathrm{~nm} \mathrm{Ag}$ sphere isolated (upper panel) and on a 70-nm-thick Ag buffer (lower panel), in a plane containing the $E$-field propagation direction $(x)$ and the $E$-field polarization $(y)$. 
an isolated $75 \mathrm{~nm}$ diameter $\mathrm{Ag}$ sphere and for the same sphere touching the 70-nm-high Ag substrate. The reported 2D maps display "hot" regions with an enhancement ranging between 1 and 100; while the LFEF calculated near the surface of the isolated sphere ( 1 or $2 \mathrm{~nm}$ ) is approximately 50-60, the LFEF calculated around the sphere on a $\mathrm{Ag}$ substrate reaches values of 100-150. Concerning the LFEF spatial distributions, the bilobe character of the dipolar near field of a nanosphere visible in the plane containing the field polarization (Fig. 3, top panel) is preserved though with a clear perturbation owing to the substrate effects; as a consequence of the anisotropy of space, the hot LFEF isolines surrounding the sphere (Fig. 3, lower panel) are less dense on the bottom side of the nanoparticle and are spread out along the equatorial plane of the nanosphere parallel to the silver substrate.

To better characterize the field enhancement, the total field was also computed at points on a series of concentric shells (representing the emitting dipole locations) outside the silver nanosphere surface at increasing radial distances from the sphere $(5-100 \mathrm{~nm})$ (see inset of Fig. 4).

Figure 4 shows that a very large field enhancement (up to 17 for the shortest distance) is related to the interaction between the silver sphere and the silver buffer. Averaging the data in Fig. 4 over a spacer of $50 \mathrm{~nm}$ (i.e., assuming a uniform distribution of NCs), we found an average PL enhancement of 10.8 (for $d$ $=5 \mathrm{~nm}$ ). Such a large enhancement is directly responsible for the measured MEF. The key point here is that the field enhancement is not only present close to the sphere, as in an isolated system or on a dielectric substrate [3], but is also spread in a region distant from the thin silver layer. We note also that silver buffer alone gives a significant PL enhancement $[2,6,8]$ (we measured a factor of 6.1 for $d=5 \mathrm{~nm}$ ). Owing to the low coverage of the MNPs, we found, from a single geometrical analysis, that for $d=5 \mathrm{~nm}$ only about $60 \%$ of the NCs in the organic layer can feel the LFEF of the MNPs, while the others can be considered interacting with the thin silver buffer only. We

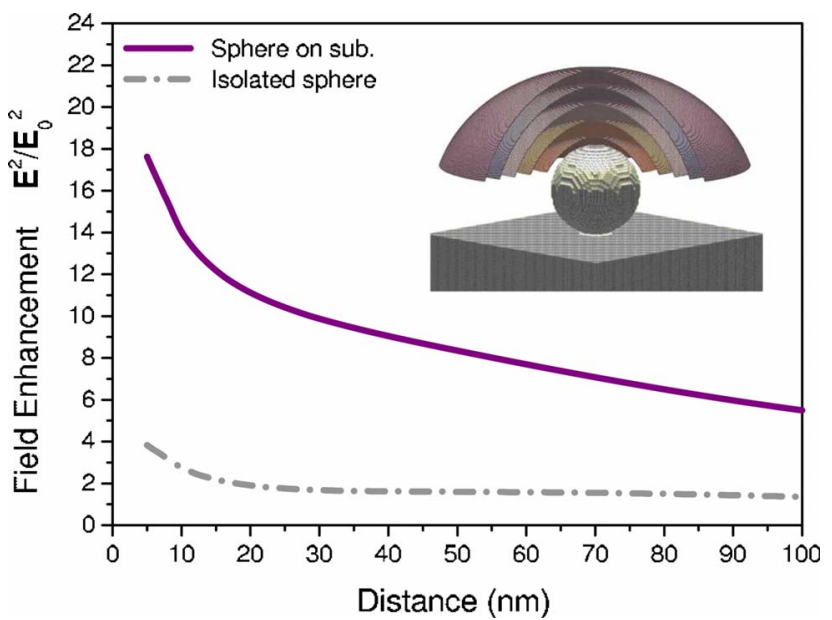

Fig. 4. (Color online) Averaged (on a 1-nm-thick virtual shells surrounding the sphere) LFEF for a $75 \mathrm{~nm}$ Ag sphere isolated (dashed-dotted curve) and on a $70 \mathrm{~nm}$ thick $\mathrm{Ag}$ buffer (solid curve), for different radial distances. thus obtain $0.6 \times 10.8+0.4 \times 6.1 \approx 9$, in good agreement with the experiment.

In conclusion, we have performed accurate electromagnetic calculations of a silver nanosphere on a thin silver buffer, including retardation effects and an approach to remove finite-substrate effects, which we believe to be novel. We found a broad region of large LFEF, which is related to the interaction of localized plasmons of the nanospheres with the underlying thin silver layer. Such broad LFEF distribution rationalizes the fluorescence enhancement from emitting dipoles embedded in an organic film observed in PL measurements.

The authors acknowledge the Iniziativa Calcolo Parallelo 2008 by CINECA consortium and CNRINFM. This work was partially supported by the European Research Council (ERC) Starting Grant, Project DEDOM (207441), and the Ministero dell'Istruzione, dell'Università e della Ricerca (MIUR) FIRB project (RBLA03ER38). R. J. Phaneuf acknowledges support from a National Science Foundation (NSF) international grant (OISE0242579) and from NSF-MRSEC (DMR-0080008).

\section{References}

1. S. A. Maier, Plasmonics: Fundamentals and Applications (Springer Science-Business Media LLC, 2007).

2. E. Fort and S. Grésillon, J. Phys. D 41, 013001 (2008).

3. S.-H. Guo, S.-J. Tsai, H.-C. Kan, D.-H. Tsai, M. R. Zachariah, and R. J. Phaneuf, Adv. Mater. 20, 1424 (2008).

4. P. P. Pompa, L. Martiradonna, A. Della Torre, F. Della Sala, L. Manna, M. De Vittorio, F. Calabi, R. Cingolani, and R. Rinaldi, Nat. Nanotechnol. 1, 126 (2006).

5. S. Sabella, G. Vecchio, R. Cingolani, R. Rinaldi, and P. P. Pompa, Langmuir 24, 13266 (2008).

6. Y. S. Chi, H. R. Byon, B. S. Lee, B. Kong, H. C. Choi, and I. S. Choi, Adv. Funct. Mater. 18, 3395 (2008).

7. H. R. Stuart and D. G. Hall, Phys. Rev. Lett. 80, 5663 (1998).

8. E. G. Matveeva, I. Gryczynski, A. Barnett, Z. Leonenko, J. R. Lakowicz, and Z. Gryczynski, Anal. Biochem. 363, 239 (2007).

9. J. Cesario, M. U. Gonzalez, S. Cheylan, W. L. Barnes, S. Enoch, and R. Quidant, Opt. Express 15, 10533 (2007).

10. V. P. Drachev, M. D. Thoreson, V. Nashine, E. N. Khaliullin, D. Ben-Amotz, V. J. Davisson, and V. M. Shalaev, J. Raman Spectrosc. 36, 648 (2005).

11. K. L. Kelly, E. Coronado, L. L. Zhao, and G. C. Schatz, J. Phys. Chem. B 107, 668 (2003).

12. C. Noguez, J. Phys. Chem. C 111, 3806 (2007).

13. M. Paulus, P. Gay-Balmaz, and O. J. Martin, Phys. Rev. E 62, 5797 (2000).

14. P. I. Geshev, U. C. Fisher, and H. Fuchs, Opt. Express 15, 13796 (2007).

15. B. T. Draine and P. J. Flatau, J. Opt. Soc. Am. A 11, 1491 (1994).

16. M. A. Yurkin and A. G. Hoekstra, J. Quant. Spectrosc. Radiat. Transf. 106, 558 (2007).

17. M. Duval Malinsky, K. L. Kelly, G. C. Schatz, and R. P. Van Duyne, J. Phys. Chem. B 105, 2343 (2001).

18. M. A. Yurkin and A. G. Hoekstra, Amsterdam Discrete Dipole Approximation, available online at http:// www.science.uva.nl/research/scs/Software/adda. 